\title{
E-CDGM: An Evolutionary Call-Dependency Graph Modularization Approach for Software Systems
}

\author{
Habib Izadkhah ${ }^{1}$, Islam Elgedawy ${ }^{2}$, Ayaz Isazadeh ${ }^{1}$ \\ ${ }^{1}$ Department of Computer Science, Faculty of Mathematical Sciences, University of Tabriz, Tabriz, \\ Iran \\ ${ }^{2}$ Department of Computer Engineering, Middle East Technical University, Northern Cyprus Campus, \\ Guzelyurt, Mersin 10, Turkey \\ Emails:izadkhah@tabrizu.ac.irelgedawy@metu.edu.trisazadeh@tabrizu.ac.ir
}

\begin{abstract}
Lack of up-to-date software documentation hinders the software evolution and maintenance processes, as simply the outdated software structure and code could be easily misunderstood. One approach to overcoming such problems is using software modularization, in which the software architecture is extracted from the available source code; such that developers can assess the reconstructed architecture against the required changes. Unfortunately, existing software modularization approaches are not accurate, as they ignore polymorphic calls among system modules. Furthermore, they are tightly coupled to the used programming language. To overcome such problems, this paper proposes the E-CDGM approach. E-CDGM decouples the extracted call dependency graph from the programming language by using the proposed intermediate code language (known as $m C o d e$ ). It also takes into consideration the polymorphic calls during the call dependency graph generation. It uses a new evolutionary optimization approach to find the best modularization option; adopting reward and penalty functions. Finally, it uses statistical analysis to build a final consolidated modularization model using different generated modularization solutions. Experimental results show that the proposed E-CDGM approach provides more accurate results when compared against existing well-known modularization approaches.
\end{abstract}

Keywords: E-CDGM, call-dependency graph, software architecture, modularization, evolutionary approach.

\section{Introduction}

Software architecture provides developers with the higher-level structural information necessary for comprehending software systems, as the architecture model provides information about the system components, as well as their 
interconnections and interfaces [1]. During the software maintenance and evolution processes, the actual software architecture could deviate from the originally documented architecture. Such architecture changes are not necessarily well documented, and in some extreme cases are not documented at all, as in legacy software systems. This of course leads to software miscomprehension, which hinders the software future evolution and maintenance processes. Hence, developers who want to understand the system opt to manually study the source code to recreate the system architecture. Of course, the manual approach does not work when the software system is quite big and has complex relationships between its modules. Hence, numerous attempts have been continuously made to design automated or semi-automated software architecture extraction tools [2]. Such automated approach for software architecture extraction is known as software modularization, which is a key activity in the reverse engineering process adopted to improve software understanding and maintenance [3-5]. The goal of the software modularization process is to automatically partition the classes of a software system into modules (or subsystems, i.e., a number of interrelated classes), such that the connections between the classes of different modules (called coupling) are minimized, and the connections between the classes of the same module (called cohesion) are maximized. In general, low coupling and high cohesion are famous characteristics for well-designed software systems [6,7], as they have a significant impact on critical software quality attributes such as reliability, portability, reusability, operability, flexibility, testability and maintainability [2].

The first step in the software modularization process is to extract a Call Dependency Graph (CDG) from the source code. A CDG indicates the method invocations between software's classes, details are given in Section 2. This step should take into consideration different relation types between classes such as method-to-method calls, class-in-method definitions, aggregation, namespace, polymorphic calls and static classes. After the CDG extraction, it should be modularized to extract the appropriate architecture. Unfortunately, existing approaches for CDG generation such as $[1,8]$ are pessimistic, that they tend to generate big sizes CDGs regardless of the required design semantics, which of course have a negative impact on the quality of the resulting modularization. Nevertheless, they ignore important design aspects such as polymorphic calls. Furthermore, they are tightly coupled to the used programming language. To overcome these problems, this paper proposes the E-CDGM (Evolutionary Call Dependency Graph Modularization) approach. E-CDGM extracts the CDG from the given source code, and decouples the extracted CDG from the programming language by using the proposed intermediate code language (known as mCode). This intermediate code language converts the source code of any language into mCode; removing any unnecessary details. The generated mCode is used to generate the CDG; taking into consideration method-method calls, class-method definitions, aggregation, namespace, polymorphic calls and static classes, details are given in Section 4. The E-CDGM approach uses a new evolutionary optimization approach to find the best modularization option; adopting both reward and penalty functions, which increases the speed of the modularization process. Finally, it uses 
statistical analysis to build a final consolidated modularization model from different optimized generated modularization solutions. Experimental results show that the proposed E-CDGM approach provides more accurate results when compared against existing well-known modularization approaches.

The rest of the paper is organized as follows. Section 2 provides the background information required for CDG extraction, and addresses the limitations of the existing works. Section 3 discusses the proposed intermediate code language and its semantics, While Section 4 explains the proposed algorithm for CDG extraction. Section 5 proposes the new evolutionary approach for CDG modularization, while Section 6 provides the statistical analysis approach used to build the final consolidated modularization model. Section 7 provides the E-CDGM evaluation experiments. Finally, Section 8 concludes the paper.

\section{Background and related work}

This section provides the basic information required for CDG extraction. The main aim of the CDG is to provide an abstract view of software system structure [1]. A CDG indicates the method invocations between software's classes. For example, consider the following sample code:

class B \{

Public A o;

Public void $\mathrm{m} 1()\{\mathrm{o} . \mathrm{m}() ;\}$;

\}

In this code, a call like o.m() inside class B, in which "o" is an object of class A clearly creates a dependency between the two classes A and B, through a method in class B.

Let $\left\{N_{1}, N_{2}, \ldots, N_{8}\right\}$ denotes a software system including eight classes; Fig. 1 shows a sample CDG this software system.

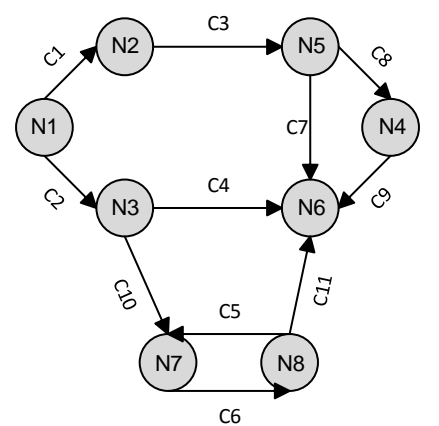

Fig. 1. A sample call dependency graph

In object oriented programming languages, each node of the CDG represents a class and the edges represent a method call between two classes of the source code, i.e., if node 1 and node 2 show class 1 and class 2 respectively, the edge between them represents a method call, i.e., in the Class 1, a (public) method of Class 2 is 
called. In a CDG, there can also be weighted edges. This way, the weight of an edge indicates the number of relationships between classes.

Currently, there are many tools for CDG extraction such as Acacia (for $\mathrm{C}^{++}$ programs) [9], Columbus (for $\mathrm{C}^{++}$programs) [8], Chava (for Java programs) [10], NDepend (www.ndepend.com; for most object oriented programs), Understand (www.scitools.com; for most object oriented programs), Bauhaus (for most object oriented programs) [11], DPMld (for .Net platform) [12] Reveal (for $\mathrm{C}^{++}$programs) [13] and Imagix-4D (www.imagix.com; for most object oriented programs). Unfortunately, these tools are tightly coupled to the adopted programming language, furthermore they (excluding DPMld) identify only inheritance, aggregation, and instantiation relationships, and totally ignores the polymorphic calls when creating CDGs. To show such limitation, let us consider the pseudo codes in Figs 2 and 3. Code in Fig. 2 does not include polymorphic call, while code in Fig. 3 does.

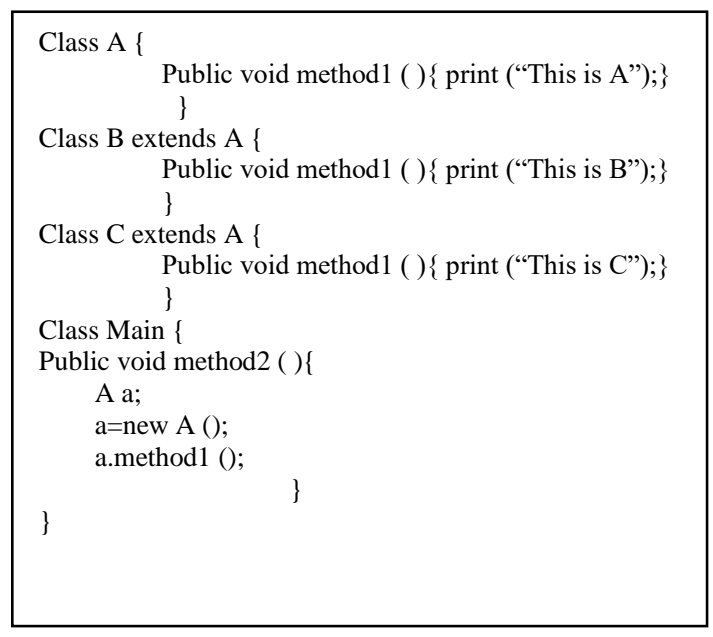

Fig. 2. A pseudo code without polymorphic call

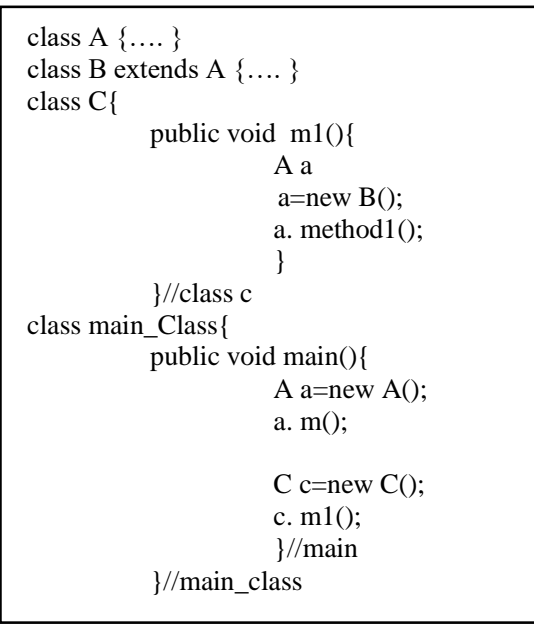

Fig. 3. A pseudo code includes a polymorphic call

In Fig. 2, the declared type for variable " $a$ " is class "A" and "a" instantiated of class "A". Thus call destination a.method 1() is considered class "A". While in Fig. 3, in class "C", the declared type of "a" is class A but "a" instantiated of class "B". Thus, call destination a.method1( ) should be considered class "B" not class "A". This kind of call is called polymorphic call. Fig. 4 shows CDG generated for Fig. 3 by existing Chava, NDepend and Understand tools, while the appropriate CDG for Fig. 3 is shown in Fig. 5. Existing tools construct the call graph pessimistically that they conservatively do not eliminate any probable call from the graph. As a result, the obtained call graph will have so many edges and a negative impact on the modularization result, as the computation of coupling and cohesion metrics will not be precise. In such cases, existing tools consider both classes as call destination. 


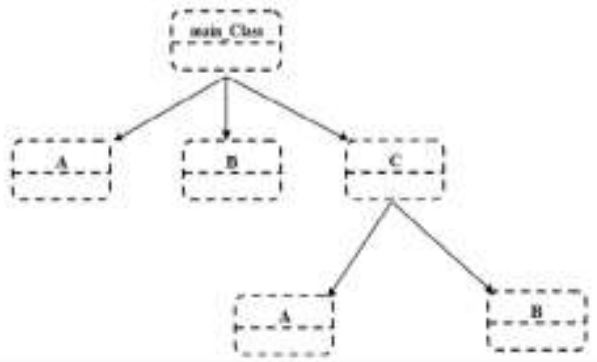

Fig. 4. CDG generated for Fig. 3 by Chava, NDepend, Understand, and Bauhaus algorithms

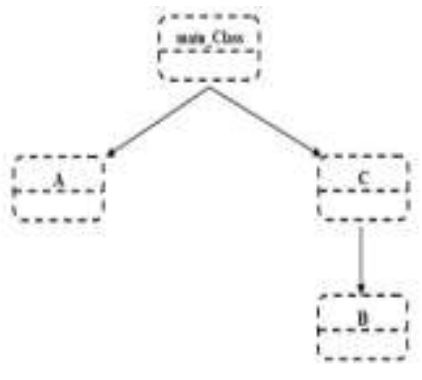

Fig. 5. Appropriate CDG for Fig. 3

Modularization of a software system is NP hard problem, particularly if input state space is very large. Hence, heuristic techniques such as genetic algorithm are adopted for finding the optimal or near optimal answer during a reasonable time such as the works in [14-22]. Genetic modularization algorithms are very subjective [1, 17], and adopted by well-known tools such as Bunch [1], Dagc [17] and Craft [18]. Bunch is a well-known tool for software modularization, and widely used in industry. Efficient behaviour of a genetic algorithm depends on proper design of encoding. One of the disadvantages of the Bunch algorithm is the largeness of search space due to presence of some repetitive answers, i.e., generated codes that have apparently different representations, but in reality, they represent the same partition. Search space in Bunch algorithm and some existing algorithms is $n^{n}$; this large search space decelerates speed of this algorithm to find appropriate architecture. E-CDGM overcomes such problem by using reward and penalty functions, and by building a consolidated model from different modularization answers appeared in the search space. Bunch and CRAFT algorithms use Acacia and Chava algorithms to construct CDG. Therefore, these tools are tightly coupled to $\mathrm{C}^{++}$and java programming languages. Also, DAGC algorithm use FRTA algorithm to construct $\mathrm{CDG}$, which is tightly coupled to the java programming language; therefore, other programming languages cannot use DAGC features. E-CDGM overcomes this problem by using the intermediate code language mCode to generate the CDGs.

\section{The proposed intermediate code language (mCode)}

The purpose of the intermediate code language is to decouple the CDG extraction process from the adopted programming language. Hence, the source code written in any language will be converted into the intermediate code language known as the mCode, where the generated intermediate code consists of information extracted from the source code that will be used as an abstract model for CDG extraction. To achieve such goal, the lexical structure of the programming language as well as the source code will be given as inputs to the mCode convertor. Fig. 6 shows the adopted conversion process.

The Flex complier is a tool that automatically generates the lexical analyzer. This tool takes the lexical specification of source language then produces the related 
lexical analyser, which will take the source code as input to automatically generate the intermediate code. The programming language lexical specifications are manually constructed according to the proposed intermediate code semantics shown in Table 1. It is important to note that the programming language lexical specifications are defined only once, and it will be used later for any java programs to convert them into mCode.

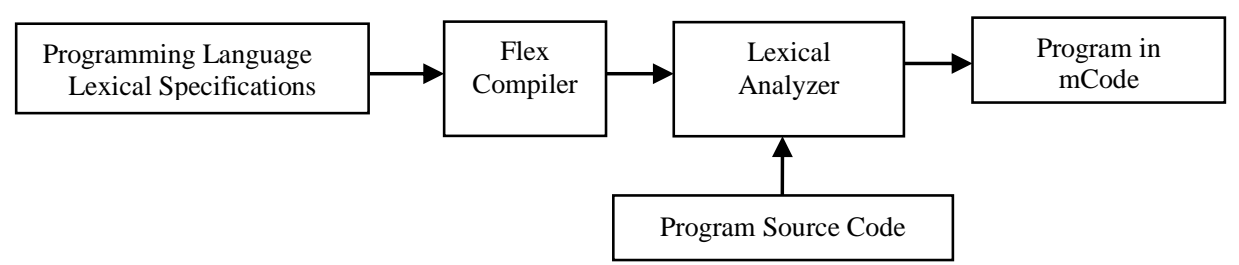

Fig. 6. Generation steps of intermediate code

Every intermediate code is described, based on a meta-model that indicates some facts that should be extracted from the source code. This meta-model is extracted and saved in a descriptive language named mCode. According to the meta-model in Table 1, the extracted intermediate-model includes class, interface, attributes of class, method of class, parameters of methods, inheritance relation, abstract, the relation of instantiated a class, call and access to attribute of a class. In Table 1, the command structures in mCode have the format: Opcode $\operatorname{par}_{1}, \operatorname{par}_{2}, \ldots$, $\operatorname{par}_{n}$, where Opcode represents one of the programming language contexts (i.e., commands and keywords), and par ${ }_{i}$ represents an attribute describing the involved context. For example, in java source code, the context of "Class" can be an Opcode and its describing attributes are class name, namespace. Hence, this command in our intermediate model is written as: Class namespace, class name. Table 1 shows the mCode Opcodes and their parameters.

Table 1. Opcode formats in mCode

\begin{tabular}{|c|c|c|}
\hline Intermediate code opcode & Description & Comment \\
\hline $\begin{array}{c}\text { Class } \\
\text { \{namespace.\}[ClassName }]\{: \text { Type }\}\end{array}$ & $\begin{array}{c}\text { Define a class in a specific } \\
\text { namespace (or package in java) }\end{array}$ & $\begin{array}{l}\text { Type: abstract, interface, } \\
\text { static, sealed, ... }\end{array}$ \\
\hline Struct \{namespace.\}[StructName] & $\begin{array}{l}\text { Define a struct in a specific } \\
\text { namespace }\end{array}$ & \\
\hline Begin [BlockType] & Begin of BlockType & $\begin{array}{l}\text { Block type indicate the } \\
\text { Namespace, Struct or Class }\end{array}$ \\
\hline End [BlockType] & End of BlockType & \\
\hline $\begin{array}{c}\text { Inherits }\left\{\begin{array}{c}\text { namespace. }\}[\text { ClassName }]\{: \\
\text { AccessType }\}\end{array}\right.\end{array}$ & Identify the class inherited from & $\begin{array}{l}\text { Access type can be as } \\
\text { public, protected or private }\end{array}$ \\
\hline $\begin{array}{c}\text { Field } \\
{[\text { FieldName]:\{namespace. }\}[\text { ClassName }} \\
]\end{array}$ & $\begin{array}{l}\text { Class include field of other class as } \\
\text { ClassName }\end{array}$ & \\
\hline Method [MethodName] $\{:$ Type $\}$ & Identify a method within a class & Type: static, ... \\
\hline $\begin{array}{c}\text { Parameters } \\
{[\text { [name]:\{namespace. }\}[\text { ClassName] }}\end{array}$ & Identify parameters of a method & \\
\hline Returns \{namespace.\}[ClassName] & $\begin{array}{l}\text { Identify the return value of a class } \\
\text { that are as class }\end{array}$ & \\
\hline $\begin{array}{c}\text { Call } \\
\{\text { namespace. }\}\{\text { ClassName. }\}[\text { Name }]\end{array}$ & Identify call variable (object) & \\
\hline $\begin{array}{c}\text { Var } \\
\text { [VarName]:\{namespace. }\}[\text { ClassName] }\end{array}$ & $\begin{array}{c}\text { Define a variable type of } \\
\text { ClassName }\end{array}$ & \\
\hline $\begin{array}{c}\text { Property } \\
{[\text { PropertyName:VarType }]\{: \text { Type }\}}\end{array}$ & Property of type of VarType & Type: static, ... \\
\hline
\end{tabular}


Fig. 7 shows the lexical specification for the java programming language using the proposed mCode semantics. This lexical specification is used to generate the mCode for any java program. For example, the source code given in Fig. 8 will be converted into the mCode depicted in Fig. 9.

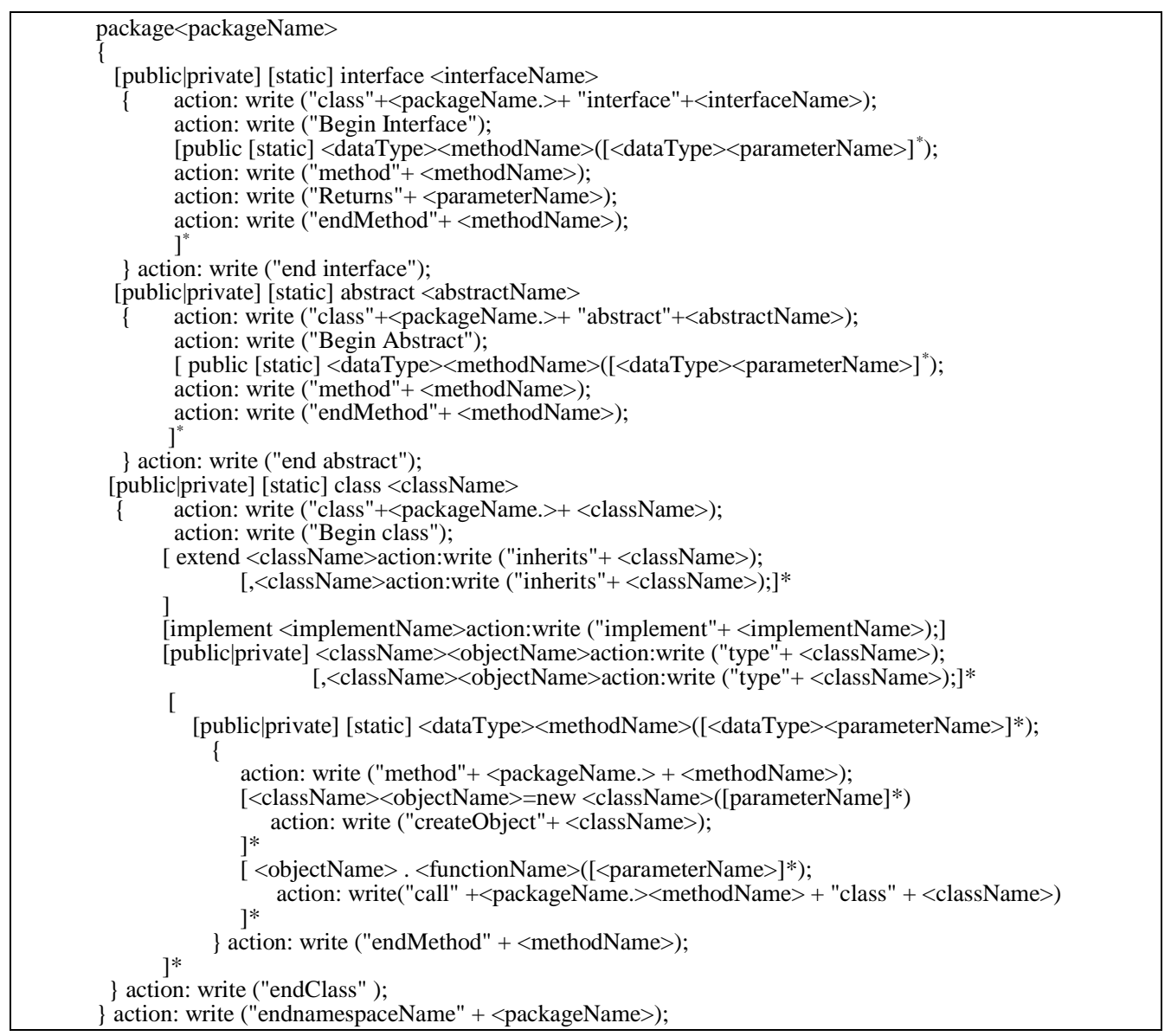

Fig. 7. Java lexical specification using mCode

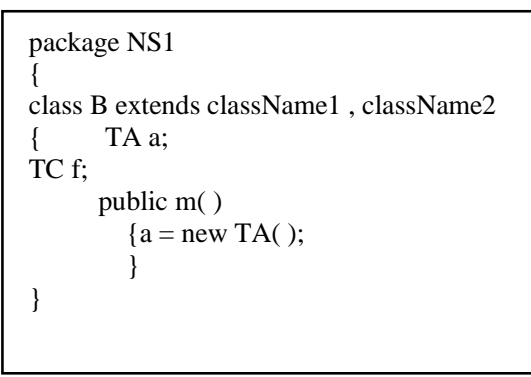

Fig. 8. A sample source code

\begin{tabular}{|c|c|}
\hline Class NS1 B & \\
\hline Begin class & \\
\hline Inherits className1 & // inherits class B from \\
\hline Inherits className2 & // inherits class B from \\
\hline Type TA // a variab & le of class $A$ in classB \\
\hline Type TC// a variable & of class $C$ in class $B$ \\
\hline Method $\mathrm{m} / /$ a metho & $\mathrm{d}$ named $\mathrm{B}$ in class $\mathrm{B}$ \\
\hline Begin method & \\
\hline Call TA & \\
\hline EndMethod $\mathrm{m}$ & //end of method \\
\hline EndClass B & //end of class \\
\hline endNamespace NS1 & // end of name space \\
\hline
\end{tabular}

Fig. 9. The generated intermediate code for Fig. 8 


\section{The proposed CDG extraction approach}

In this section, we propose a new algorithm to generate CDG from the intermediate code considering the type of relation between classes such as, method-method, class-method, aggregation, namespace, polymorphic calls and static class. In general, classes are related with each of the following two ways.

1. Interaction Type. Determines ways in which the two classes communicate with each other. M.

- Aggregation: are of the form class-attribute as a class D is the field of class

- Class-method: in this case, class D is the type of a parameter of method $m_{\mathrm{C}}$ of a class $\mathrm{C}$, or if a class $\mathrm{D}$ is the return type of method $m_{\mathrm{C}}$.

- Method-method: in this case, method $m_{\mathrm{D}}$ of a class D directly invokes a method $m_{\mathrm{C}}$ of a class $\mathrm{C}$, or a method $m_{\mathrm{D}}$ receives via parameter a pointer to $m_{\mathrm{C}}$ thereby invoking $m_{\mathrm{C}}$ indirectly

2. Relation Type. Determines ways in which the two classes are related to each other.

- Inheritance: in this case, class D inherits attribute and behaviour of class $\mathrm{C}$ or vice versa.

- Friendship: in this case, a friend class to have access to the private and protected members of the class.

- Other relations between classes $\mathrm{C}$ and $\mathrm{D}$ are interface and abstract.

Variable Type Analysis (VTA) [23] algorithm is a well-known algorithm for determining destination of a call that is used in compiler construction. We recast it for constructing CDG in software modularization domain. In this section, we extend VTA to support static classes and name spaces, and then we explain how to construct precise CDG from the generated intermediate code (including explicit and polymorphic calls). The aim of the enhanced VTA is to precisely determine a call's destination.

Definition 1. Destination of a call such as o.m(), in this algorithm showed as Destination(o), it is identified as follows:

a) If call of $\mathrm{o}$ has a declared class type $\mathrm{C}$, the possible run-time of $\mathrm{o}$, Destination(o), includes $\mathrm{C}$ and all sub-classes of $\mathrm{C}$.

b) If call of $o$ has a declared interface $I$, the possible run-time of $o$, Destination(o), includes: (1) the set of all classes that implement $I$ or implement a sub interface of $I$, which we call implements $(I)$; (2) all subclasses of implements $(I)$.

The main aim is to identify a set of reaching variable to o in each call likes o.m( ) precisely. This set, called Receiving-types(o). The proposed algorithm uses a graph to perform this action. For example, we say type A reaches to variable o if once at least there would be one path in the program run to be started by object of type A (e.g., as $v=$ new $\mathrm{A}())$, and then chain of assignment would be as follows:

$$
x_{1}=v, x_{2}=x_{1}, \ldots, x_{n}=x_{n-1}, \mathrm{o}=x_{n} .
$$

Each one of the assignments would be a call or return value of a method. Given a program mCode, CDG is constructed using algorithm 1 (i.e., the enhanced VTA algorithm). The algorithm has five main steps. The first step is about 
constructing the CDG graph using the variables and the assignments. The second step is about revising the graph based on the inheritance relations. The third step is about removing cycles from the graph. The forth step is about computing the possible receiving nodes for each call to check type propagations. Finally, the fifth step is about determining the actual destination of each call.

In Fig. 10a we give the important parts of an example program. Fig. 10b to Fig. 10e show above Steps 1-4 of Algorithm 1 for code in Fig 10a. Fig. 10b shows construction of the graph based on assignments in code. That in the source code if we have $\mathrm{a} 1=\mathrm{a} 2$, in this case, in the constructed graph, we will an edge from a2 to a1 and so on. Fig. 10c shows the instantiated class of variables (i.e., the initial assigned values), for example, in Fig. 10a, we have a1= new $\mathrm{A}()$; therefore, in the Fig. 10c the label of a1 is $\{A\}$, and we have $b 1=$ new $B()$ then the label of $b 1$ is $\{B\}$ and so on. Fig. 10d shows removal of cycles from graph that if some of variables are located in cycle, and they have no type, in this case we consider them as a node. Fig. 10e shows propagation of types. As nodes a 3 and b3 are in a cycle, hence they are converted to a united node before propagation. After calculating Receivingtypes (o) set for each call using Algorithm 1, the actual destination of each call is determined using Equation 2.

Algorithm 1. Enhanced VTA for determining actual destination of a call

Input: The program mCode

Output: The extracted CDG

Step 1. Graph Construction, in which nodes show variables and each edge as $a \rightarrow b$ shows an assignment as $b=a$.

Step 1.1. Nodes are created as follows:

1) for each field $f$ (where $f$ has a reference to a class) in class $\mathrm{C}$ into namespace NS, creates a new node labelled with NS.C. $f$ aggregation

// This condition occurs when a class is defined as static class or occurs

2) for each method $m$ in class $C$ into namespace NS, creates a new node labelled NS.C.m

Step 1.2. Edges are added as follows:

For each statement of form lhs=rhs; or lhs $=(\mathrm{C})$ rhs; where lhs and rhs must be an ordinary, field or array reference, we add a directed edge from the rhs node to the lhs node.

Step 2. Initialized graph, in which all assignment would be searched as lhs=new type and type would be placed as initial value in Receiving-types(lhs) set.

Step 3. Remove all cycles from the graph and generate a new directed graph without cycles. To remove cycles, the nodes those are located in a cycle to be converted into a node. Receiving-types (lhs) of this node would be obtained from the union of nodes.

Step 4. Compute the Receiving-types(o) set for each call through propagation of types in the graph.

Step 5. After above works, actual destination of each call, EIMA(o), would be obtained by following relation:

$$
\operatorname{EIMA}(\mathrm{o})=\operatorname{Destination}(\mathrm{o}) \cap \text { Receiving-types }(\mathrm{o}) \text {. }
$$




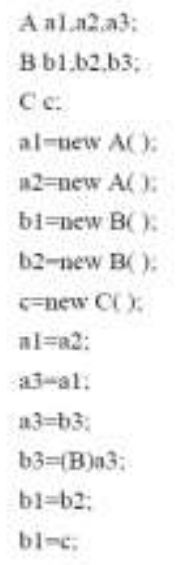

(a)
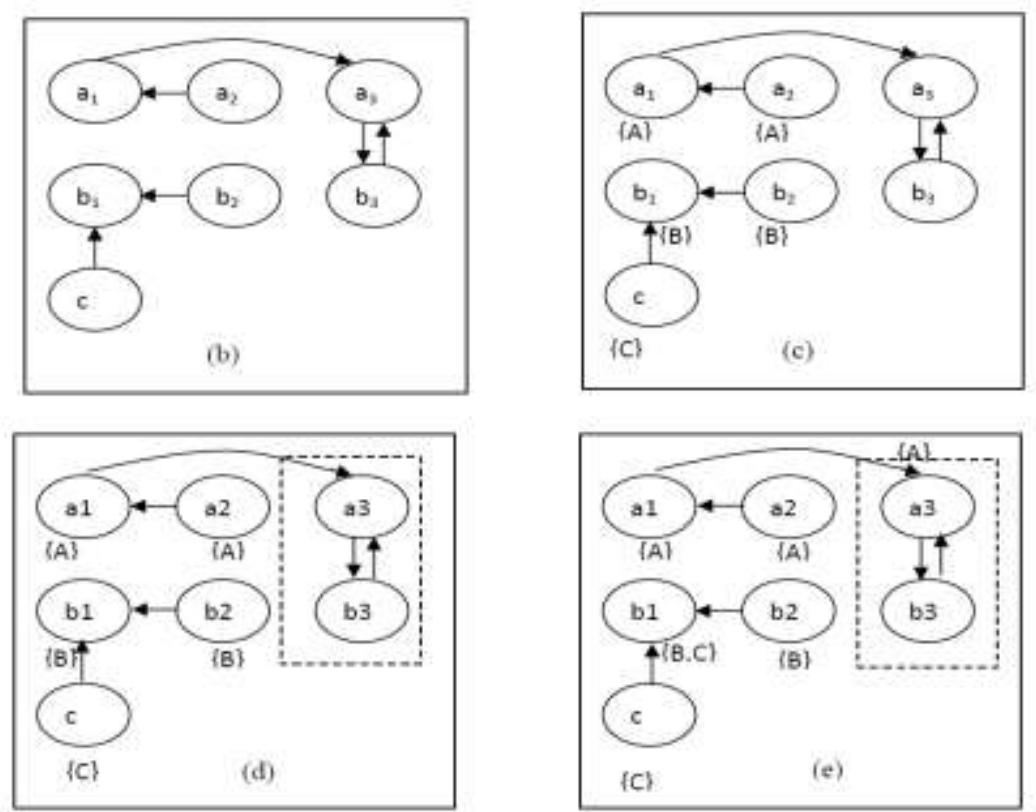

Fig. 10. Computing the Receiving-types(o) set for each call

\section{The proposed CDG modularization approach}

The general problem of graph partitioning (of which software modularizing is a special case) is NP-hard [1]. Therefore, to reduce the time complexity to a polynomial upper bound, most researchers using heuristic based algorithms for software modularizing. In this section, we propose a new evolutionary algorithm to modularize software systems. First, we will discuss the proposed encoding scheme, and then go on to discuss the used fitness, reward, and penalty functions. Finally, we discussed the proposed evolutionary algorithm for CDG modularization.

\subsection{The proposed modularization encoding approach}

Each modularization solution is encoded as a vector (i.e., a learning automaton) and each vector represents a permutation of nodes of the CDG. The number of vector cells is the number of CDG classes. Each vector cell includes four rows, where the first row is the class number (i.e., $m$ ), the second row is the partner number of a class (i.e., $p$ ), the third row is the depth of cell vector (this required in learning) and the fourth row is the selection probability of each class for penalty or reward. The initial selection probabilities for the classes are equal (as shown in Fig. 11). Each vector's cell is called an action. The partner number of a class is any class number in the CDG that has the potential to be included with the class number $m$ in the same module. The partner number is determined according to the numbering method proposed in [17], in which if the partner number $p$ for class $m$ be equal or greater than $m$, then $m$ is placed in a new module; otherwise $m$ belongs to the same 
module that $p$ is allocated in that. Once the partners of every cell are defined, modules could be determined by grouping all related partners into the same module. For example, Fig. 11 shows a given CDG and its corresponding vector structure. As we have 6 classes, then we will have 6 cells, every cell is assigned a partner, for instance the partner for class 2 is class 5 , and the partner for class 3 is class 6 , while class 1 has no partners in this case it is assigned to itself. Once the partners' allocation is finalized, we can see we can partition the CDG into three modules, module 1 has only class 1 , while module 2 has classes 2 and 5 , and finally module 3 has classes 3, 4, and 6 .

This efficient encoding reduces number of permutations from $n^{n}$ to $n$ !. This reduction in size of search space would result in faster convergence of the algorithm.

\begin{tabular}{|c|c|c|c|c|c|c|}
\hline $\begin{array}{c}\text { Class number } \\
(m)\end{array}$ & 1 & 2 & 3 & 4 & 5 & 6 \\
\hline$P$ & 1 & 5 & 6 & 3 & 2 & 4 \\
\hline Depth & 0 & 0 & 0 & 0 & 0 & 0 \\
\hline Probability & 0.16 & 0.16 & 0.16 & 0.16 & 0.16 & 0.16 \\
\hline
\end{tabular}

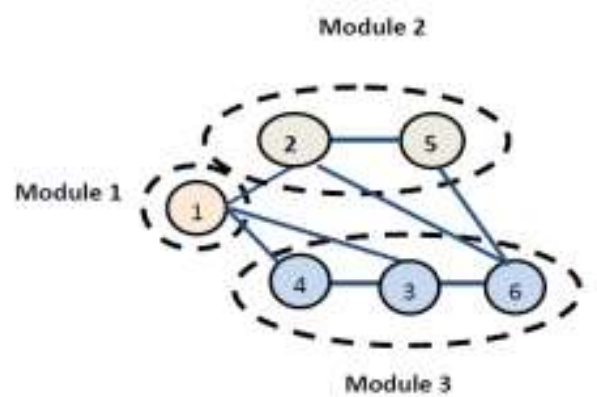

Fig. 11. A CDG partition and its corresponding Vector structure

A vector is defined as tuple $\{a, v, \beta, \varphi, F, P, T\}$ in which:

- $a=\left\{a_{1}, \ldots, a_{r}\right\}$ is a set of vector's actions ( $r$ is number of the software classes)

- $v=\left\{v_{1}, \ldots, v_{r}\right\}$ is a set of used objects in the vector. These objects do not include module number of graph nodes; they are other node numbers of graph. These objects moving in various situations of vector and produce different permutation (objects are shown in Fig. 11 by the name of $p$.)

- $\beta=\left\{\beta_{1}, \ldots, \beta_{r}\right\}$ is the result of evaluation of a selected action. If $\beta_{i}=0$, i.e., selected action meets the desired criteria, it should be rewarded. If $\beta_{i}=1$, i.e., selected action does not meet the desired criteria, it should be penalized.

- $\varphi_{1}, \varphi_{2}, \ldots, \varphi_{R N}$ is set of situations; $N$ is the number of states an action can go through to decide a mutation is needed or not; $R$ is the number of vector actions. 
- $F: \varphi \times \beta \rightarrow \varphi$ is mapping method of situations. This method determines the next situation from $\beta$ value and current situation.

- $P=\left\{p_{1}, \ldots, p_{r}\right\}$ is probabilities array. This array shows selection probability of each action and then upon either rewarding or penalty would change after each selection. For action $i$, the action probability is

$$
P_{i}(t)=\frac{1}{r}, i=1,2, \ldots, r \quad(r \text { is the number of classes }) ;
$$

- $T=T[a(n), \beta(n), p(n)]$ is learning algorithm (described in Section 6.4).

\subsection{The adopted vector fitness function}

Quality function is used to determine the fitness degree of each vector in population. Our aim to modularize is to increase cohesion and decrease coupling of modules as much as possible. Thus, we adapt quality function presented in [1] to consider the cases mentioned earlier. Suppose:

$C_{1}=$ class-attribute and $\left|C_{1}\right|=$ number of class-attributes in the source code, $w_{1}=$ weight of $C_{1}$

$C_{2}=$ class-method and $\left|C_{2}\right|=$ number of class-methods in the source code, $w_{2}=$ weight of $C_{2}$

$C_{3}=$ method-method and $\left|C_{3}\right|=$ number of method-methods in the source code, $w_{3}=$ weight of $C_{3}$

We define the quality function for each generated module as follows:

(4)

$$
\mathrm{MF}_{m}=\frac{2\left(\sum_{i=1}^{3} w_{i}\left|C_{i}\right|\right)}{2\left(\sum_{i=1}^{3} w_{i}\left|C_{i}\right|\right)+\sum_{k=1}^{3}\left(w_{k} \sum_{j=1, j \neq i}^{\text {\#modules }}\left(\left|C_{i, j}\right|+\left|C_{j, i}\right|\right)\right)}, 1 \leq m \leq \text { number of modules in a vector, }
$$

$\left|C_{i, j}\right|$ represents the call numbers from module $i$ to module $j$ and $\left|C_{j, i}\right|$ represents the call numbers from module $j$ to module $i$. For module $m(1 \leq m \leq k)$, where $k$ is the number of modules, the Module Factor, $\mathrm{MF}_{m}$, is a normalized ratio between the total weight of the internal edges (edges within the module) and half of the total weight of external edges (edges that exit or enter the module). The Modularization Quality (MQ) for a CDG partitioned into $k$ modules is calculated by summing the Module Factor (MF) for each module:

$$
\mathrm{MQ}=\sum_{m=1}^{k} \mathrm{MF}_{m}
$$

\subsection{The proposed reward and penalty functions}

The evolutionary process of proposed algorithm is accelerated using learning. In the proposed algorithm, the learning is done using reward and penalty functions. For this purpose, beside evaluation of vectors, the actions are evaluated based on its effect on vector value. So, the most proper location for actions inside vectors is gradually determined during the evolutionary process. Generally, penalty and reward are applied in the proposed algorithm in this manner: During modularization 
process, the algorithm selects action $a_{i}$ in a vector and evaluates it, if it receives favourable response $\left(\beta_{i}=0\right)$, probability $\left(P_{i}(n)\right)$ related to this action would increase and probability of other actions would decrease. If it receives an unfavourable response $\left(\beta_{i}=1\right), P_{i}(n)$ related to this action would decrease and the probability of other actions would increase. In this paper, we use the linear learning scheme proposed in [24], which computes the linear learning scheme for multiple actions as follows:

$$
\begin{gathered}
f_{j}\left[p_{j}(n)\right]=a p_{j}(n), \quad 0<a<1, \\
g_{j}\left[p_{j(n)}\right]=\frac{b}{r-1}-b p_{j(n)} .
\end{gathered}
$$

Functions $g_{j}$ and $f_{j}$ are non-negative functions, which are called reward and penalty functions, respectively. In above equations $r, a$, and $b$ are respectively number of actions in a vector, reward and penalty parameters. We can control rate of convergence of a vector by setting $a$ and $b$ parameters. In the Equations (6) and (7), the learning algorithm is known as linear reward penalty if $a$ and $b$ are equal. If $b$ is much smaller than $a$, the learning algorithm is known as linear reward epsilon penalty. Penalized and reward probability functions in linear learning algorithms are defined as follows:

For a favourable response $i$ :

$$
p_{j}(n+1)=p_{j}(n)-f_{j}\left[p_{j}(n)\right] \quad \forall j, j \neq i,,
$$

but

and

$$
f_{j}\left[p_{j}(n)\right]=a p_{j}(n) ; \text { so, } p_{j}(n+1)=p_{j}(n)-a p_{j}(n)=(1-a) p_{j}(n),
$$

$$
\begin{gathered}
p_{i}(n+1)=p_{i}(n)+\sum_{j=1, j \neq 1}^{r} f_{j}\left[p_{j}(n)\right]= \\
=p_{i}(n)+\sum_{j=1, j \neq i} a p_{j}(n)=p_{i}(n)+a \sum p_{j}(n)=p_{i}(n)+a\left(1-p_{i}(n)\right) .
\end{gathered}
$$

Unfavourable response $i$

$$
p_{j}(n+1)=p_{j}(n)+g_{j}\left[p_{j}(n)\right],
$$

but

$$
g_{j}\left(p_{j}(n)\right)=\frac{b}{r-1}-b p_{j}(n),
$$

so

and

$$
p_{j}(n+1)=p_{j}(n)+\frac{b}{r-1}-b p_{j}(n)=\frac{b}{r-1}+(1-b) p_{j}(n),
$$

$$
\begin{gathered}
p_{i}(n+1)=p_{i}(n)-\sum_{j=1, j \neq i}^{r} g_{j}\left[p_{j}(n)\right]_{=} \\
=p_{i}(n)-\sum\left(\frac{b}{r-1}-b p_{j}(n)\right)=p_{i}(n)-b+b \sum p_{j}(n)=p_{i}(n)-b+b\left(1-p_{i}(n)\right)= \\
=p_{i}(n)-b+b-b p_{i}(n)=(1-b) p_{i}(n), \quad 0 \leq b<1 .
\end{gathered}
$$




\section{Algorithm 2. Evaluation of an action of a vector for doing reward and penalty}

Step 1. Select an action of a vector according to its probability (Equations (8)(11))

// an action of a vector indicates a vertex in CDG

Step 2. Compute vertex cohesion

//Vertex cohesion is the ratio of number of vertices connected to vertex "u" inside the module containing this vertex to the total number of intra-connections this module //

Step 3. Compute vertex coupling

//Vertex coupling is the ratio of number of inter-connections vertices connected to vertex $u$ to the total number of inter-connections vertices possible to be connected to this module//

Step 4. If (vertex cohesion - vertex coupling > $\frac{\mathrm{MQ}}{K}$ )

//where $K$ represents number of modules in vector and MQ is defined in Equation (5)

Step 4.1. The vertex will be rewarded

Step 5. Else

Step 5.1. The vertex is penalized // the modularization is not appropriate

The main aim of these probabilities is to use previous behaviour of the system in order to take decisions for the future, hence, learning occurs. In each repetition of the evolutionary modularization algorithm, an action of each vector would be selected according to its probability (as in Equations (8)-(11) and this action can be evaluated as in Algorithm 2.

The modularization algorithm selects an action $a_{i}$ in a vector based on its probability (Equations (8)-(11) and evaluates it (Algorithm 2). If number of unfavourable responses of an action were more than number of favourable responses, this action would be replaced by another action to generate a new permutation.

6.4. The proposed evolutionary modularization algorithm

The modularization algorithm takes the following inputs:

1) The number of vectors to be generated $|V|$. It is the number of possible modularization solutions to be generated at a given time.

2) The vectors maximum depth $N$. It represents the number of states an action can go through to decide its mutation. It can be seen as the number of internal states an action can go through during the learning process.

3) The number of generations to be done $\boldsymbol{G}$. Any generated vector could be mutated to search for better solutions, however to avoid having an infinite number of mutation, we specify a maximum number of generations a vector can go through.

Based on the given number of vectors, several vectors are generated randomly. The algorithm performs following steps on all vectors until the given number of generations is reached. The modularization algorithm selects an action $a_{i}$ in a vector 
based on its probability, and then evaluates it as in Algorithm 2. Based on the evaluation results, it will decide to keep the action in its place in the modularization solution, or change its place to find a better modularization solution (i.e., perform a mutation operation). The decision of an action mutation is decided based on the internal state the action has, as we do not want to perform a mutation step every time an action is penalized. To explain this idea let us assume a vector includes $R$ actions $\left(\left\{a_{1}, a_{2}, a_{3}, \ldots, a_{R}\right\}\right)$ and has $R N$ internal state $\left(\varphi_{1}, \varphi_{2}, \ldots, \varphi_{R N}\right)$. Internal states of $\varphi_{1}, \varphi_{2}, \ldots, \varphi_{N}$ are related to $a_{1} ; \varphi_{N+1}, \varphi_{N+2}, \ldots, \varphi_{2 N}$ are related to $a_{2}$, and

$\varphi_{(R-1) N+1}, \varphi_{(R-1) N+2}, \ldots, \varphi_{R N}$ are related to $a_{R} ; \varphi_{1}$ represents the deepest state for $a_{1}$; and $\varphi_{N}$ is the most shallow state for $a_{1}$, similarly $\varphi_{N+1}$ represents the deepest state for $a_{2}$, and $\varphi_{2 N}$ is the most shallow state for $a_{2}$, and so on. For example, if we let $N=5$, it means that each state machine has 5 states, so 5 (i.e., the shallowest state) to 1 (i.e., the deepest state) for action 1, while states 10 (i.e., the shallowest state) to 6 (i.e., the deepest state) are for action 2. Hence, $N$ is border-state of the first action and $2 N$ is border-state of the second action, and so on. Every action will start at a given state, and it will move inwards towards deeper states if it is rewarded, and it will move outwards towards shallower state if it is penalized. If an action reaches a border-state and receives undesirable response, it would be displaced by another action in the vector, in other words, a mutation is need and a new permutation of classes and modules would be generated. Jumping between actions, means moving from the shallowest of the penalized action, to the shallowest state of the next action. The algorithm searches for an action in the vector for displacement so that MQ value in that permutation is more than others. If MQ value of new permutations generated is lower than initial permutation, it remains the same initial permutation. The proposed modularization algorithm is shown in Algorithm 3.

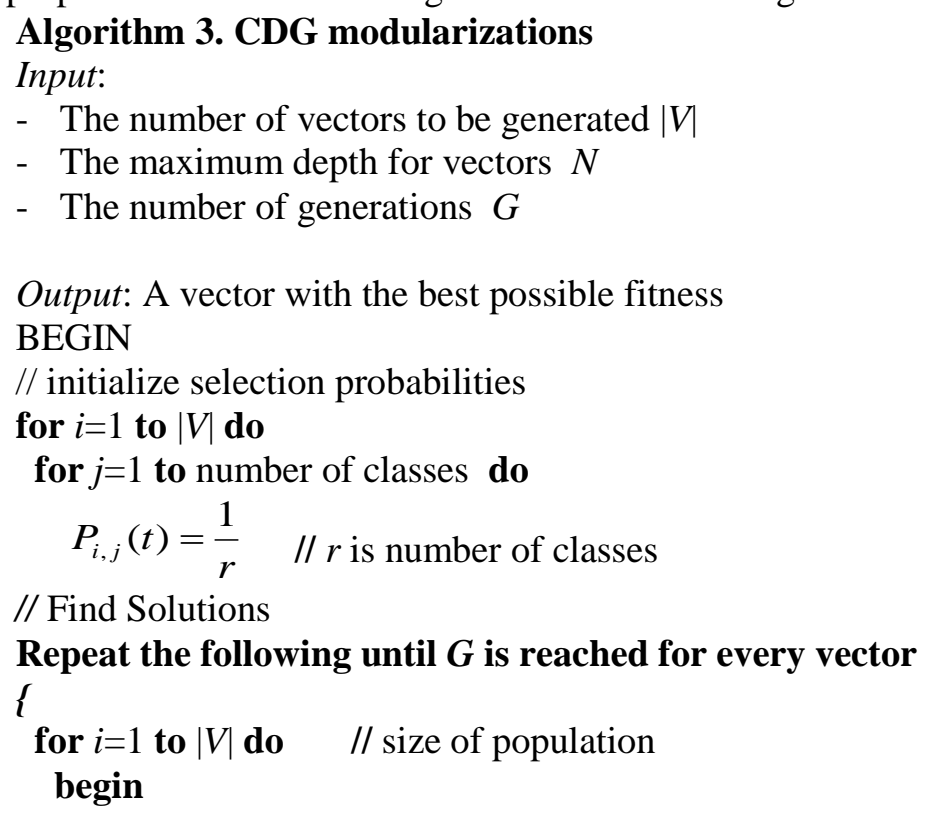


- Select Action $_{\mathrm{u}}$ of the Vector $_{i}$ with probability $P_{i}(t)$

- IF vertexCohesion $\left(\right.$ Action $\left._{u}\right)-$ vertexCoupling $\left(\right.$ Action $\left._{u}\right) \geq \frac{\mathrm{MQ}}{k}$ THEN

$/ / k$ is the number of modules

begin

- reward (Action $)$ ); //Make inwards to a deeper state

-Update its probability using Equations (8) and (9)

end;

ELSE

begin

If Action $_{u}$ is in border-state and It is Penalized)

for $i=1$ to number of actions in vector $i$ do

begin

- Action ${\text { would be displaced randomly with } \text { Action }_{T} \text { in vector }}_{i}$

- Produce a new permutation as vector ${ }_{j}$

- if $\left(\mathrm{MQ}\left(\right.\right.$ vector $\left._{j}\right) \geq \mathrm{MQ}\left(\right.$ vector $\left.\left._{i}\right)\right)$

Accept a new permutation and return the new permutation Else

// return the new vector

vector $_{i}$ remains in the same previous permutation end;

Else

begin

Penalize $(u) ; \quad$ // move outwards to a shallower state

Update its probability using Equations (10) and (11)

end;

end; // ELSE

end;// for

\}

END.

\section{The proposed modularization consolidation approach}

Due to the heuristic nature of proposed algorithm in Section 6, it may produce results with the same quality but different modularization for different runs on a given graph. In different modularization solutions, it is observed regular displacement of several classes between different modules, while other classes displace less. Hence, we believe the common patterns between the obtained solutions should appear in the final solution. To achieve this aim, we calculate the percentage that two different classes are placed in the same module in different obtained modularizations, then using statistical analysis to decide if they should appear together in the final solution or not. The statistical analysis is done as follows. 
Let $C=\left\{C_{1}, C_{2}, \ldots, C_{n}\right\}$ is the set of classes in CDG. In each repetition of graph modularization process, modularization result represented as $\left\{C_{i}, C_{j}\right\}$ relations is saved in one file which two classes of $C_{i}$ and $C_{j}$ are placed in same module. We then derive a tuple of $\left\{C_{i}, C_{j}, f\right\}$ (that represents $\mathrm{f}$ times of $C_{i}$ and $C_{j}$ in different modularization is placed in same module) and show them as set $D$. For example, suppose we have modulated the call graph in Fig. 12 for three solutions, and the results of these modularizations are in Fig. 13. Fig. 14 shows the unified model for Fig. 13.

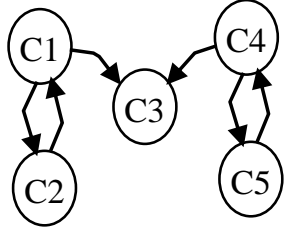

Fig. 12. A sample CDG

\begin{tabular}{|ccc|}
\hline$\{\mathbf{C} 1, \mathbf{C} 2\}$ & $\{\mathbf{C} 1, \mathbf{C} 2\}$ & $\{\mathbf{C} 1, \mathbf{C} 2\}$ \\
$\{\mathrm{C} 4, \mathrm{C} 5\}$ & $\{\mathrm{C} 4, \mathrm{C} 5\}$ & $\{\mathrm{C} 4, \mathrm{C} 5\}$ \\
$\{\mathrm{C} 1, \mathrm{C} 3\}$ & $\{\mathrm{C} 1, \mathrm{C} 3\}$ & $\{\mathrm{C} 3, \mathrm{C} 4\}$ \\
$\{\mathrm{C} 2, \mathrm{C} 3\}$ & $\{\mathrm{C} 2, \mathrm{C} 3\}$ & $\{\mathrm{C} 3, \mathrm{C} 5\}$ \\
Modularization 1 & Modularization 2 & Modularization 3 \\
\hline
\end{tabular}

Fig. 13. Obtained modularization from modularizing of CDG in Fig. 12
$\{\mathrm{C} 1, \mathrm{C} 2,3\}$ $\{\mathrm{C} 4, \mathrm{C} 5,3\}$

$\{\mathrm{C} 1, \mathrm{C} 3,2\}$

$\{\mathrm{C} 2, \mathrm{C} 3,2\}$

$\{\mathrm{C} 3, \mathrm{C} 4,1\}$

$\{\mathrm{C} 3, \mathrm{C} 5,1\}$

Fig. 14. The unified model for Fig. 13

Finally, to create common consolidated model we derive tuples of $\mathrm{D}$ which $f$ is higher or equal to threshold $\theta$ and then place that in same module. The problem now, what is the suitable value of the threshold $\theta$ to be used, as the final result accuracy totally depends on it? We will determine the threshold value empirically using real life data for real life systems, as shown in Section 7.1.

\subsection{Empirical computation for the consolidation threshold $\theta$}

To identify the appropriate value for threshold $\theta$ for creating a common model, we analyze three real life large-scale software systems, whose original modularizations are available. We compute different modularization solutions for these systems for different values of the threshold $\theta$, and then choose the best threshold values that provide the most similar modularizations to the original architectures.

The adopted software systems are as follows: Compost (http://www.thecompost-system.org, 2003) library with 32 JAVA packages and up to 500 classes, Apache-ant (http://ant.apache.org, 2004) which contains a set of java packages for web server and finally a library called Swing (http://javasoft, 2002). For abovementioned software systems, the generated call dependency graph is modularized several times by proposed modularization algorithm. For each modularization, MQ and architecture similarities to original architecture is calculated. Similarity criteria are computed based on the harmonic mean of Precision/Recall. This criterion measures the similarity between two modularizations based on co-modules pairs in modularizations.

The call dependency graph of the Compost library is modularized 14 times by the proposed algorithm and each modularization result is compared to original modularization. Table 2 shows these results. Clearly, although MQ of most of the modularizations are quite the same, percentage of similarities is in large-scale diverse compared to original structure. 
Table 2. Evaluating modularization results $\left(\mathrm{P} / \mathrm{R}=\right.$ Precision/Recall, $F_{m}=$ Harmonic Mean of P/R)

\begin{tabular}{|c|c|c|c|c|c|c|c|c|c|c|c|c|c|c|}
\hline Resulr & 1 & 2 & 3 & 4 & 5 & 6 & 7 & 8 & 9 & 10 & 11 & 12 & 13 & 14 \\
\hline P/R 66 & $45 / 27$ & $39: 20$ & $26 / 15$ & $46: 51$ & $45[27$ & $34 / 12$ & $26 / 12$ & 3817 & $22 / 9$ & $36 / 18$ & $24 / 11$ & 2310 & $36 / 4 !$ & 4435 \\
\hline$F_{m}$ & 33,75 & 26.44 & 19.02 & 48.37 & 33.75 & 17.73 & 16,42 & 23.49 & 12.77 & 24.0 & 1508 & 13,93 & 47.34 & 3898 \\
\hline $\begin{array}{c}\text { Modularization } \\
\text { Quality }\end{array}$ & 19.1 & 19.8 & 18.2 & 19.0 & 18.8 & 182 & 17.0 & 199 & 191 & 28.7 & 18.3 & 18.0 & 18.3 & 185 \\
\hline
\end{tabular}

Table 3 shows the abovementioned modularization results and confidence analysis for different threshold values. Table 3 shows high percentage of similarities of threshold value from 50 up to 60 . Obviously, if we decrease threshold value lower than $50, F_{m}$ would decrease significantly. If we increase threshold value higher than 50, e.g., 80 and 90, we expect percentage of similarities to be higher, but it is not, as the original architecture is not optimum (maximum cohesion and minimum coupling).

Table 3. Variation of $F$ for different thresholds for Compost

\begin{tabular}{|l|c|c|c|c|c|c|c|c|c|c|}
\hline Threshold $(\theta)$ & 90 & 80 & 70 & 60 & 50 & 40 & 30 & 20 & 10 & 1 \\
\hline Precision & 21 & 20 & 18 & 54 & 54 & 41 & 18 & 3 & 3 & 2 \\
\hline Recall & 1 & 1 & 2 & 44 & 24 & 22 & 56 & 69 & 71 & 74 \\
\hline$F_{m}$ & 1.82 & 1.82 & 3.2 & 48.48 & 33.23 & 28.63 & 27.24 & 5.75 & 5.75 & 3.89 \\
\hline
\end{tabular}

To prove that the obtained threshold range provides better consolidation quality; we computed the similarity of the consolidated model for the three above mentioned systems using a threshold value as $60 \%$. Table 4 shows the highest similarity value of an obtained individual solution against the similarity of the common model. As it was expected, the created consolidated model is the most similar to the original architecture when compared with individual modularization solutions for every system.

\section{E-CDGM evaluation experiments}

In this section, we compare the experimental results obtained of the proposed E-CDGM algorithm and two well-known algorithms such as Bunch and DAGC. We will use different data sets for testing these algorithms both artificial datasets and real-life data sets. Since in Bunch and DAGC the quality of the modules is computed by TurboMQ function [1, 18]; MQ used in this paper is different compared to TurboMQ. In TurboMQ equation, the types of relations among classes are not considered; in other words, if we in MQ (i.e., Equation (4)) set $w_{i}=1$ and considers the type of relation between two methods as method-method, in this case, the MQ will be same TurboMQ, so we can use the TurboMQ to compare between the algorithms.

Table 4. Comparison results

\begin{tabular}{|l|c|c|c|}
\hline Model & Swing & Apache & Compost \\
\hline Highest obtained individual solution similarity & $41 \%$ & $35 \%$ & $43 \%$ \\
\hline Consolidated model similarity & $61 \%$ & $57 \%$ & $49 \%$ \\
\hline
\end{tabular}

To compare E-CDGM, with Bunch and DAGC, first we tested the algorithms using artificial data set, in which seven different CDGs with more than 200 nodes 
are used. Each CDG was modularized twenty times. The average results are shown in Table 5.

As we can see, E-CDGM performs the best, as it creates a consolidated model from the different obtained solutions, while other approaches just return individual solutions. To see if E-CDGM still performs better than other algorithms, we compare them using real life data with characteristics shown in Table 6. Table 7 shows the final quality value of the modularization solutions obtained by E-CDGM, DAGC, and Bunch. Results of this table is the best result among 20 times algorithm run at the same execution time period (i.e., $100 \mathrm{~s}$ ). As we can see in Table 7, E-CDGM still performs better than the other algorithms for the same allowed execution period. This confirms our claims that using a consolidated model to generate the final solution always provides the best results.

Table 5. Comparing quality of results with TurboMQ function

\begin{tabular}{|c|c|c|c|c|c|c|c|}
\hline \multicolumn{7}{|c|}{ Modularization quality (TurboMQ) } \\
\hline Number of nodes & 200 & 250 & 300 & 350 & 400 & 450 & 500 \\
\hline Bunch & 6.15 & 6.22 & 7.67 & 8.90 & 5.89 & 6.0 & 6.30 \\
\hline DAGC & 6.90 & 6.95 & 8.0 & 8.87 & 6.93 & 7.53 & 7.20 \\
\hline E-CDGM & 7.10 & 7.35 & 9.12 & 9.98 & 7.20 & 8.93 & 9.20 \\
\hline
\end{tabular}

Table 6. Real-life data sets and their characteristics

\begin{tabular}{|c|c|c|c|}
\hline $\begin{array}{c}\text { Software } \\
\text { systems }\end{array}$ & $\begin{array}{c}\text { Number of system } \\
\text { modules }\end{array}$ & $\begin{array}{c}\text { Number of relation } \\
\text { between modules }\end{array}$ & System description \\
\hline Compiler & 13 & 32 & Turing compiler \\
\hline Boxer & 18 & 29 & Graph design system \\
\hline Mini tunis & 20 & 57 & Operation system \\
\hline Ispell & 24 & 103 & Unix spell checking \\
\hline
\end{tabular}

Table 7. Comparing quality of results with TurboMQ objective function

\begin{tabular}{|c|c|c|c|c|c|c|}
\hline Algorithm & \multicolumn{2}{|c|}{ Bunch [1] } & \multicolumn{2}{c|}{ DAGC [17] } & \multicolumn{2}{c|}{ E-CDGM } \\
\hline $\begin{array}{c}\text { Software } \\
\text { systems }\end{array}$ & $\begin{array}{c}\text { TurboMQ } \\
\text { quality }\end{array}$ & Time, s & $\begin{array}{c}\text { TurboMQ } \\
\text { quality }\end{array}$ & Time, s & $\begin{array}{c}\text { TurboMQ } \\
\text { quality }\end{array}$ & Time, s \\
\hline Compiler & 1.42 & 100 & 1.65 & 100 & 1.91 & 100 \\
\hline Boxer & 2.81 & 100 & 2.92 & 100 & 2.99 & 100 \\
\hline Mini tunis & 2.21 & 100 & 2.28 & 100 & 2.49 & 100 \\
\hline Ispell & 1.95 & 100 & 2.09 & 100 & 2.41 & 100 \\
\hline
\end{tabular}

\section{Conclusion}

In this paper, we proposed a new approach for software modularization known as E-CDGM (Evolutionary Call Dependency Graph Modularization). E-CDGM generates a call dependency graph from the given source code. It decouples the extracted call dependency graph from the programming language by using the proposed intermediate code language (known as mCode). It also takes into consideration the polymorphic calls during the call dependency graph generation. It uses a new evolutionary optimization approach to find the best modularization option; adopting reward and penalty functions. Finally, it uses statistical analysis to build a final consolidated modularization model using different generated 
modularization options. Consolidation aggregation threshold is determined empirically to be in the range of (0.5-0.6). Experimental results show that the proposed E-CDGM approach provides more accurate results when compared against existing well-known modularization approaches.

\section{References}

1. Mitche11, B. S., S. Mancoridis. On the Automatic Modularization of Software Systems Using the Bunch Tool. - Software Engineering, IEEE Transactions On, Vol. 32, 2006, No 3, pp. 193-208.

2. Q if e n g, Z., Q. D e h o n g, T. Q u b o, S. L e i. Object-Oriented Software Architecture Recovery Using a New Hybrid Clustering Algorithm. - In: 7th International Conference on Fuzzy Systems and Knowledge Discovery (FSKD'2010), 2010, pp. 2546-2550.

3. Lung, C., M. Z a man, A. Nandi. Applications of Clustering Techniques to Software Partitioning, Recovery and Restructuring. - Journal of Systems and Software, Vol. 73, 2004, pp. 227-244.

4. Lung, C., X. Xu, M. Z a man, A. S rinivas a n. Program Restructuring Using Clustering Techniques. - The Journal of Systems and Software, Vol. 79, 2006, pp. 1261-1279.

5. B ittencourt, R. A., G. D. D. S erey. Comparison of Graph Clustering Algorithms for Recovering Software Architecture Module Views. - In: Proc. of Software Maintenance and Reengineering (CSMR'2009), IEEE Computer Society Press, pp. 251-254.

6. Pre s s m a n, R. S. Software Engineering: A Practitioner's Approach. Eighth Ed. 2014, McGrawHill, Inc.

7. Poshyva ny k, D., A. M a r cus, R. Fere n c, T. Gy i mót hy. Using Information Retrieval based Coupling Measures for Impact Analysis. - Empirical Software Engineering, Vol. 14, 2009, No 1, pp. 5-32.

8. Fer e n c, R., A. B e s z e d e s, T. G y i m ó th y. Extracting Facts with Columbus from $\mathrm{C}^{++}$Code. - In: Proc. of the Eighth CSMR, 2004, pp. 4-8.

9. Chen, Y., E. Gansner, E. Kouts ofios. A $\mathrm{C}^{++}$Data Model Supporting Reachability Analysis and Dead Code Detection. - In: Proc. of 6th European Software Engineering Conf. and 5th ACM SIGSOFT Symposioum on the Foundations of Software Engineering, 1997.

10. Korn, J., Y. Chen, E. Kouts ofios. Chava: Reverse Engineering and Tracking of Java Applets. - In: Proc. of Working Conf. on Reverse Engineering, 1999.

11. R a z a, A., G. V o g e l, E. P 1 öd e r e de r. Bauhaus - A Tool Suite for Program Analysis and Reverse Engineering. - In: Reliable Software Technologies. Ada, Europe 2006, pp. 71-83.

12. Dobiš, M., L. U. Majtás. Mining Design Patterns from Existing Projects Using Static and RunTime Analysis. - In: Software Engineering Techniques. Berlin, Heidelberg, Springer, 2008, pp. 62-75.

13. C 1 a r k e, P. J., T. H. Gib b s, B. A. Ma 11 o y, J. F. Power. Reveal: A Tool to Reverse Engineer Class Diagrams. - In: Proc. of 14th International Conference on Tools Pacific: Objects for Internet, Mobile and Embedded Applications CRPIT'2002, Sarah Matzko, pp. 13-21.

14. M k a ou er, W., M. Kes sentini, A. S h o ut, P. Kolighe u, S. B e chikh, K. De b, A. O u n i. Many-Objective Software Remodularization Using NSGA-III. - ACM Transactions on Software Engineering and Methodology (TOSEM), Vol. 24, 2015, No 3, p. 17.

15. B a vota, G., F. Carnevale, A. De Lucia, M. Di Penta, R. Oliveto. Putting the Developer in-the-Loop: An Interactive GA for Software Re-Modularization. - In: Search Based Software Engineering, Berlin, Heidelberg, Springer, 2012, pp. 75-89.

16. B avota, G., A. De Lucia, A. Marcus, R. Oliveto. Using Structural and Semantic Measures to Improve Software Modularization. - Empirical Software Engineering, Vol. 18, 2013, No 5, pp. 901-932.

17. Parsa, S., O. Bushehrian. A New Encoding Scheme and a Framework to Investigate Genetic Clustering Algorithms. - Journal of Research and Practice in Information Technology, Vol. 37, 2005, No 1, pp. 127-143. 
18. M it c h e 11, B. S., S. M a n c or i d i s. CRAFT: A Framework for Evaluating Software Clustering Results in the Absence of Benchmark Decomposition. - In: Proc. of IWPC, IEEE, 2001.

19. R ä i ä, O. A Survey on Search-Based Software Design. - Computer Science Review, Vol. 4, 2010, Issue 4, pp. 203-249.

20. Harman, M., S. A. Ansouri, J. Zhang. Search Based Software Engineering: A Comprehensive Review. Technical Report TR-09-03, 2009, King's College, London, United Kingdom.

21. A u f f a r th, B. Clustering by a Genetic Algorithm with Biased Mutation Operator. - WCCI CEC, IEEE, 2010, pp. 18-23.

22. M a magh a n i, A., M. R. Me ybodi. Clustering of Software Systems Using New Hybrid Algorithms. - In: Proc. of IEEE 11th International Conference on Computer and Information Technology, 2009, pp. 20-26.

23. S und aresan, V., L. Hendre n, C. R a z a Fi mahefa, R. V allé e-Rai, P. La m, E. Ga g n o n, C. G o d i n. Practical Virtual Method Call Resolution for Java. - ACM, Vol. 35, 2000, No 10, pp. 264-280.

24. B u s h, R. R., F. M o s t e 11 e r. Stochastic Models for Learning. New York, Wiley, 1958. 